\title{
Comparison of existing poroelastic models for wave damping in a porous seabed
}

\author{
M. Lin ${ }^{a}$, D.S. Jeng ${ }^{b, *}$ \\ ${ }^{a}$ Institute of Applied Mechanics, Chinese Academy of Science, Beijing 100080, China \\ b School of Engineering, Griffith University Gold Coast Campus, Queensland 9726, Australia
}

Received 3 June 2002; accepted 25 September 2002

\begin{abstract}
Mechanism of wave-seabed interaction has been extensively studied by coastal geotechnical engineers in recent years. Numerous poro-elastic models have been proposed to investigate the mechanism of wave propagation on a seabed in the past. The existing poro-elastic models include drained model, consolidation model, Coulomb-damping model, and full dynamic model. However, to date, the difference between the existing models is unclear. In this paper, the fully dynamic poro-elastic model for the wave-seabed interaction will be derived first. Then, the existing models will be reduced from the proposed fully dynamic model. Based on the numerical comparisons, the applicable range of each model is also clarified for the engineering practice.
\end{abstract}

(C) 2002 Elsevier Science Ltd. All rights reserved.

Keywords: Consolidation model; Coulomb friction; Drained behaviour; Pore pressure; Effective stresses

\section{Introduction}

Ocean and lakebeds are usually covered with various types of sediments, such as gravel, coarse sand, fine sand, clay and so on. As waves propagate on the seabed, the wave energy is transmitted into the marine sediments, and wave profile is modified due to the sediments motion and percolation. However, most water-wave theories being used in coastal or offshore design assume the seabed to be a rigid and impervious medium, which is inevitable to bring some error.

\footnotetext{
* Corresponding author. Tel.: +617-5552-8683; fax: +617-5552-8065.
}

E-mail address: d.jeng@griffith.edu.au (D.S. Jeng). 
Many processes are responsible for water wave attenuation, such as seepage flow forced through the pores of the bed, the associated friction on the walls of the internal pores. To date, there are two methods to handle the problems. The first method is to treat marine sediment as a single phase material. For example, Gade (1958) and Dalrymple and Liu (1978) assumed soft sediment behaves as a viscous fluid. Hsiao and Shemdin (1980) and Macpherson (1980) assumed an incompressible visco-elastic mud layer, considering the soft bed to have the property of a Voigt body. However, the assumption of a soil as a fluid-like substance does not properly represent the nature properties of the internal friction mechanism.

The second method is to treat marine sediments as a two-phase medium: solid phase and fluid phase. Based on the assumption of a rigid and permeable sandy seabed, Reid and Kajiura (1957) may be the first to investigate the phenomenon of wave damping analytically. Later, this method has been further extended to more complicated cases (Liu, 1973; Dean and Dalrymple, 1984; Kim et al., 2000). Recently, Jeng (2001) further investigated the wave dispersion based on static Biot's elastic model. The concept was further extended to investigate the wave damping in seabed with the acceleration due to soil motion (Lee et al., 2002a). Another model of seabed dissipation is due to Coulomb-damped friction between the internal grains (Yamamoto, 1983). Based on the potential theory and Biot's theory, Yamamoto (1983) first investigated the effects of Coulomb-damped friction in an infinite seabed, and examined the non-linear mechanism for the interaction between waves and porous seabed and carried out the wave dispersion on the weak non-elastic model. This framework has been further extended to the case of finite thickness (Lin, 2001; Lee et al., 2002b).

In summary, there are three existing models to deal with the wave damping due to the wave-bed interaction with various assumptions. The differences between the models in physical significance are unclear. Furthermore, the applicable ranges of the existing models have not been clarified. In this paper, we will set out basic soil formulation to obtain a general wave dispersion and concentrate on deriving a reasonably ranges of applications of the existing models.

\section{Fully dynamic poro-elastic model}

\subsection{Boundary value problem}

The ocean wave is assumed to be propagating over porous media along X-direction, the water-soil interface is defined as the origin of the $x-z$ plane, and the $z$-axis is positive upward from the interface and negative downward. The proposed model for wave-seabed interaction is based on the potential flow theory of incompressible irrational flow for the water waves, the linearised free surface condition, and small loading wave amplitude, the velocity potential $(\varphi)$ can be written in following form:

$$
\varphi=i a_{0} g\{\cosh [\tilde{k}(z-h)] / \omega+\omega \sinh [\tilde{k}(z-h)] / g \tilde{k}\} e^{i(\varpi t-\tilde{k} x)}
$$

in which $a_{0}$ is the wave amplitude; the complex wave number is $\tilde{k}=k_{r}+i k_{i}$, the 
real part, $k_{r}$, is related to the wavelength by $k_{r}=2 \pi / L$, while $k_{i}$ is the damping of water waves; $\omega$ is the wave frequency; $h$ is the water depth, $g$ is the gravitational constant.

In this study, the seabed is considered as a porous elastic media with finite depth, homogeneous, isotropic and fully saturated, while the fluid motion is fairly well described by Darcy's law. Zienkiewicz et al. (1980) proposed a physical derivation of the governing equations, generalizing the formulation of Biot's poro-elastic theory (Biot, 1956). The equations in the form of Constitutive law, Equilibrium (overall), Equilibrium of fluid flow and Mass balance can be written as (Z-M is an abbreviation for the model):

$$
\begin{aligned}
& d \sigma_{i j}^{\prime}=D_{i j k l}\left(d \varepsilon_{k l}-d \varepsilon_{k l}^{o}\right) \\
& \sigma_{i j, j}+\rho g_{i}=\rho_{w} \ddot{w}_{i}^{\prime}+\rho \ddot{u}_{i} \\
& -p_{, i}+\rho_{w} g_{i}=\rho_{w} \ddot{w}_{i} / n_{e}+k_{i j} \dot{w}_{j}^{\prime}+\rho_{w} \ddot{u}_{i} \\
& \frac{n_{e}}{K_{w}} p+\varepsilon_{i i}+w_{i, i}^{\prime}=0
\end{aligned}
$$

where, $\sigma_{i j}=\sigma^{\prime}{ }_{i j}-\delta_{i j} p$, in which $\sigma_{i j}$ is the total stress, $\sigma^{\prime}{ }_{i j}$ denotes the effective stress, $p$ represents the pore pressure; $D_{i j k l}$ is defined by the form of the constitutive relationships; $d \varepsilon_{i j}=\left(d u_{i, j}+d u_{j, i}\right) / 2$ is strain definition, in which $u_{i}$ is the displacements of the solid matrix; $w_{i}^{\prime}=n_{e}\left(w_{i}-u_{i}\right)$ is the average relative displacement of the fluid to the solid ( $w_{i}$ is the actual pore fluid displacement), $\mathrm{n}_{\mathrm{e}}$ is the porosity of the solid phase; $\rho=\left(1-n_{e}\right) \rho_{s}+n_{e} \rho_{w}, \rho_{s}, \rho_{w}$ as the density of the solid grain and fluid respectively; $\mathrm{K}_{\mathrm{f}}$ is the bulk modulus of the fluid, represents the rate of the fluid expansion; the matrix $\mathrm{k}_{\mathrm{ij}}$ is known as the permeability matrix, in the slow flow through an isotropic medium and it is simply defined by a single value of permeability coefficient $k_{s} / \gamma=k_{s} / \rho_{w} g$. In the range of water wave, the gravity terms is very small $\left(\frac{\rho g}{\nabla p} \sim 10^{-5}\right)$, thus the gravity terms may be ignored from (2).

Based on the linear poro-elasticity, isotropic Hooke's law is assumed is given by

$$
\sigma_{i j}^{\prime}=G\left(\frac{\partial u_{i}}{\partial x_{j}}+\frac{\partial u_{j}}{\partial x_{i}}+\frac{2 \mu}{1-2 \mu} \delta_{i j} \frac{\partial u_{k}}{\partial x_{k}}\right)
$$

where $G$ is the shear modulus of the soil; $\mu$ is the Poission's ratio.

Eqs. (2a) and (2d) can be rewritten as

$$
\left\{\begin{array}{l}
G\left\{\nabla^{2} \vec{u}+\frac{1}{1-2 \mu} \nabla(\nabla \cdot \vec{u})\right\}-\nabla p=n_{e} \rho_{w} \ddot{\vec{w}}+\left(1-n_{e}\right) \rho_{s} \ddot{\vec{u}} \\
-\nabla p=\rho_{w} \ddot{\vec{w}}+\frac{\gamma n_{e}}{k_{s}}(\dot{\vec{w}}-\dot{\vec{u}}) \\
\frac{n_{e}}{K_{w}} \dot{p}+\nabla \cdot \dot{\vec{u}}-\frac{k_{s}}{\gamma} \nabla^{2} p-\rho_{w} \frac{k_{s}}{\gamma} \nabla \cdot \ddot{\vec{w}}=0
\end{array}\right.
$$




\subsection{Boundary conditions}

To solve the unknowns in (4), some appropriate boundary conditions are required. At the water-soil interface, there are four boundary conditions: the vertical effective normal stress vanishes; the fluid pressure transmits continuously and the mass of fluid must be conserved. As to the shear stresses, Sawaragi and Deguchi (1992) demonstrated that the shear stress on the interface is much smaller than other stresses, even when the loading waves are strongly non-linear. Thus, we ignore the shear stress due to wave loading at the interface. Then, the boundary conditions on the interface $\mathrm{z}=0$ are written as:

$$
z=0,\left\{\begin{array}{l}
\sigma^{\prime}=2 G\left(\frac{\partial u_{z}}{\partial z}+\frac{\mu}{1-2 \mu} \nabla \cdot \vec{u}\right)=0 \\
p=-\rho_{w} \frac{\partial \varphi}{\partial t} \\
\tau_{x z}=G\left(\frac{\partial u_{x}}{\partial z}+\frac{\partial u_{z}}{\partial x}\right)=0
\end{array}\right.
$$

and

$$
\frac{\partial \varphi}{\partial z}=\frac{\partial u_{z}}{\partial t}+\frac{\partial w_{z}}{\partial t} \quad \text { or } \quad \frac{\partial \varphi}{\partial z}=\frac{\partial u_{z}}{\partial t}-\frac{k_{s} \partial p_{s}}{\gamma \partial z}
$$

Assuming that the bottom is an impermeable and rigid, the dynamic fluctuations of all the physical quantities vanish, i.e.

$$
z=-\mathrm{d},\left\{\begin{array}{l}
u_{x}=0 \\
u_{z}=0 \\
\partial p / \partial z=0
\end{array}\right.
$$

Herein, the wave dispersion relations can be derived by the boundary condition (6) together with (1):

$$
\omega^{2}-g \tilde{k \tanh \tilde{k} h}=\frac{1}{a_{0} \cosh \tilde{k} h} \quad\left[w_{z}(0)+u_{z}(0)\right]
$$

It is clear that the right-hand-side of Eq. (8) is the complex of the vertical displacement of solid and the vertical seepage displacement, and they represent the effect of the motion and percolation of interaction on the dispersion of water waves.

\subsection{General solution}

Although Zienkiewicz et al. (1980) summarised the general governing equations of wave propagation over a porous seabed, they did not solve the two-dimensional 
problem. Alternatively, they reduced the whole problem to a one-dimensional case, and derived the analytical solution for such a case. Herein, we directly solve the problem analytically through two-dimensional analysis.

Now the boundary value problem can be solved. Due to the soil regarded as a linear elastic media, the displacement vectors of the soil can be decomposed into two parts: irrotational and non-divergent, that is

$$
\vec{u}=\nabla \phi_{s}+\nabla \times \psi_{s} \quad \vec{w}=\nabla \phi_{w}+\nabla \times \psi_{w}
$$

By substituting (9) into (4), we have

$$
\left\{\begin{array}{l}
-n_{e} \rho_{w} \ddot{\psi}_{w}-\left(1-n_{e}\right) \rho_{s} \ddot{\psi}_{s}+G \nabla^{2} \psi_{s}=0 \\
\left(1-n_{e}\right) \rho_{w} \ddot{\phi}_{w}+\frac{\gamma n_{e}}{k_{s}} \dot{\phi}_{w}-\left(1-n_{e}\right) \rho_{s} \ddot{\phi}_{s}-\frac{\gamma n_{e}}{k_{s}} \dot{\phi}_{s}+\frac{2(1-\mu)}{1-2 \mu} G \nabla^{2} \phi_{s}=0 \\
\rho_{w} \ddot{\psi}_{w}+\frac{\gamma n_{e}}{k_{s}}\left(\dot{\psi}_{w}-\dot{\psi}_{s}\right)=0 \\
\frac{n_{e}}{K_{w}} \rho_{w} \dddot{\phi}_{w}+\frac{n_{e}^{2}}{K_{w}} \frac{\gamma}{k_{s}} \ddot{\phi}_{w}-n_{e} \nabla^{2} \dot{\phi}_{w}-\frac{n_{e}^{2}}{K_{w}} \frac{\gamma}{k_{s}} \ddot{\phi}_{s}+\left(n_{e}-1\right) \nabla^{2} \dot{\phi}_{s}=0 \\
p=\frac{\gamma n_{e}}{k_{s}} \dot{\phi}_{s}-\rho_{w} \ddot{\phi}_{w}-\frac{\gamma n_{e}}{k_{s}} \dot{\phi}_{w}
\end{array}\right.
$$

Since water wave is harmonic in both time and $x$-direction, the displacement in the soil is assumed to be also harmonic in both time and $x$-direction. That is to say that $\phi_{w, s}, \psi_{w, s}$ can be expressed in terms of to $e^{i(\omega t-\tilde{k} x)}$. Putting $\phi_{w}=\tilde{c}_{1} \phi_{s}, \psi_{w}=$ $\tilde{c}_{2} \psi_{s}$, and substituting it into (10), the scalar potential and the vector potential decomposition of the soil can be expressed in the form, respectively:

$$
\left\{\begin{array}{l}
\nabla^{2} \phi_{s \pm}+\tilde{k}_{1 \pm}^{2} \phi_{s \pm}=0 \\
\nabla^{2} \psi_{s}+\tilde{k}_{2}^{2} \psi_{s}=0
\end{array}\right.
$$

where

$$
\begin{aligned}
\tilde{k}_{1 \pm}^{2}= & \frac{\left(\tilde{c}_{1 \pm}-1\right)\left[\left(n_{e}-1\right) \rho_{w} \omega^{2}-i \frac{n_{e} \gamma}{k_{s}}\right]}{G}, \tilde{k}_{2}^{2}=\frac{n_{e} \rho_{w} \tilde{c}_{2}+\left(1-n_{e}\right) \rho_{s}}{G} \omega^{2} \\
\tilde{c}_{1 \pm}= & \frac{-\tilde{A}_{4} \pm \sqrt{\tilde{A}_{4}^{2}-4 n_{e} \tilde{A}_{2}\left(\tilde{A}_{3}\left(n_{e}-1\right)-k_{p} n_{e} G^{\prime} / K_{w}\right)}}{2 n_{e} \tilde{A}_{2}}, \tilde{c}_{2}=\frac{k_{p}}{k_{p}+i \omega \rho_{w}} \\
\tilde{A}_{1} & =k_{p}+i \omega \rho_{w} \\
\tilde{A}_{2} & =k_{p}+i\left(1-n_{e}\right) \rho_{w} \omega \\
\tilde{A}_{3} & =k_{p}+i\left(1-n_{e}\right) \rho_{s} \omega \\
\tilde{A}_{4} & =\left[n_{e} G^{\prime} \tilde{A}_{1} / K_{w}-\left(n_{e}-1\right) \tilde{A}_{2}-n_{e} \tilde{A}_{3}\right] \\
k_{p} & =\frac{n_{e} \gamma}{k_{s} g} \quad G^{\prime}=G \frac{2(1-\mu)}{1-2 \mu}
\end{aligned}
$$


The general solution of (11) is expressed as:

$$
\phi_{1 \pm}=\left[\tilde{a}_{1 \pm} e^{\widetilde{k}_{1 \pm} z}+\tilde{a}_{2 \pm} e^{-\widetilde{k}_{1 \pm} z}\right] e^{i(\omega t-\tilde{k} x)}, \psi_{2}=\left[\tilde{b}_{1} e^{\widetilde{k}_{2} z}+\tilde{b}_{2} e^{-\tilde{k}_{2} z}\right] e^{i(\omega t-\tilde{k} x)}
$$

in which $\tilde{k}_{1 \pm, 2}=\tilde{k}^{2}-\tilde{k}_{1 \pm, 2}^{2}$; the six complex coefficients $\tilde{a}_{1,2( \pm)}, b_{1,2}$ can be determined from the boundary conditions (5) and (7):

$$
\left[\begin{array}{l}
\tilde{a}_{1+} \\
\tilde{a}_{1-} \\
\tilde{a}_{2+} \\
\tilde{a}_{2-} \\
\tilde{b}_{1} \\
\tilde{b}_{2}
\end{array}\right]=\tilde{A}^{-1}\left[\begin{array}{l}
0 \\
0 \\
0 \\
0 \\
\tilde{p}_{0} \\
0
\end{array}\right]
$$

where

$$
\begin{aligned}
& \tilde{A}= \\
& {\left[\begin{array}{llllll}
\tilde{\kappa}_{1+} & \tilde{k}_{1+} & \tilde{\kappa}_{1-} & \tilde{\kappa}_{1-} & i(1-2 \mu) \tilde{k} \tilde{k}_{2} & -i(1-2 \mu) \tilde{k} \tilde{k}_{2} \\
-2 \tilde{k} \tilde{k}_{1+} & 2 \tilde{k} \tilde{k}_{1+} & -2 \tilde{k} \tilde{k}_{1-} & 2 \tilde{k} \tilde{k}_{1-} & \tilde{k}_{2}^{2}+\tilde{k}^{2} & \tilde{k}_{2}^{2}+\tilde{k}^{2} \\
\tilde{k}_{1+} e^{-\tilde{k}_{1+}{ }^{d}} & -\tilde{k}_{1+} e^{\tilde{k}_{1+}{ }^{d}} & \tilde{k}_{1-} e^{-\tilde{k}_{1-} d} & -\tilde{k}_{1-} e^{\tilde{k}_{1-} d} & i \tilde{k} e^{-\tilde{k}_{2} d} & i \tilde{k} e^{\tilde{k}_{2} d} \\
-i \tilde{k}^{-\tilde{k}_{1+} d} & -i \tilde{k}^{\tilde{k}_{1+}{ }^{d}} & -i \tilde{k} e^{-\tilde{k}_{1+} d} & -i \tilde{k}^{\tilde{k}_{1+} d} & \tilde{k}_{2} e^{-\tilde{k}_{2} d} & \tilde{k}_{2} e^{\tilde{k}_{2} d} \\
\tilde{d}_{1+} & \tilde{d}_{1+} & \tilde{d}_{1-} & \tilde{d}_{1-} & 0 & 0 \\
\tilde{d}_{1+} \tilde{k}_{1+} e^{-\tilde{k}_{1+}{ }^{d}} & -\tilde{d}_{1+} \tilde{k}_{1+} e^{\tilde{k}_{1+}{ }^{d}} & \tilde{d}_{1-} \tilde{k}_{1-} e^{-\tilde{k}_{1-} d} & -\tilde{d}_{1-} \tilde{k}_{1-} e^{\tilde{k}_{1-} d} & 0 & 0
\end{array}\right]} \\
& \tilde{\kappa}_{1 \pm}=(1-\mu) \tilde{k}_{1 \pm}^{2}-\mu \tilde{k}^{2} \\
& \tilde{d}_{1 \pm}=i \frac{n_{e} \rho_{w} g}{k_{s}} \omega\left(1-\tilde{c}_{1 \pm}\right)+\rho_{w} \tilde{c}_{1 \pm} \omega^{2} \\
& \tilde{p}_{0}=\rho_{w} g\left(\cosh (\tilde{k} h)-\frac{\omega^{2}}{\tilde{k} g} \sinh (\tilde{k} h)\right)
\end{aligned}
$$

Then,

$$
u_{z}=\frac{\partial \phi_{s+}+\partial \phi_{s-}}{\partial z}-\frac{\partial \psi_{s}}{\partial x}, w_{z}=\frac{\partial \phi_{w+}+\partial \phi_{w-}}{\partial z}-\frac{\partial \psi_{w}}{\partial x}
$$

Substitute the above into (8), yielding the dispersion relation $(\tilde{k}, \omega)$

$$
\omega^{2}-g \tilde{k} \tanh (\tilde{k} h)=\left[1-\frac{\left(1+\tilde{c}_{1 \pm}\right) \phi_{s \pm}(0)-i \tilde{k}\left(1+\tilde{c}_{2}\right) \psi_{s}(0)}{a_{0} \cosh \tilde{k} h}\right]
$$




\section{Simplified models}

Note that Eq. (2) involves the basic variables $\dot{u}_{i}, \dot{w}_{i}, \ddot{u}_{i}, \ddot{w}_{i}$ and some variables can be eliminated to simplify the formulations. In this section, we will summarize three existing models, which have been widely applied in the problem of dynamic response of soil to water wave.

\subsection{Drained behaviour (D-M)}

In this model, the seabed is treated as a rigid porous media and the pore fluid is incompressible, i.e. $\dot{w}_{i}^{\prime}=0, \dot{u}_{i}=0, \ddot{w}_{i}^{\prime}=0, \ddot{u}_{i}=0$. The governing Eq. (2a)-(2d) can be rewritten as:

$$
\left\{\begin{array}{l}
d \sigma_{i j}^{\prime}=D_{i j k l}\left(d \varepsilon_{k l}-d \varepsilon_{k l}^{0}\right) \\
\sigma_{i j, j}=0 \\
-\left(k_{i j} p_{, i}\right)_{, j}=0
\end{array}\right.
$$

which leads to

$$
\nabla^{2} p=0
$$

In the model, all transient behaviour has ceased, i.e. complete uncoupling of equation occurs. Although the type of fully drained behaviour does not occur ever with dynamic effects, many investigations still applied the model to analysis interaction of water wave and structure (Dean and Dalrymple, 1984; Kim et al., 2000).

Based on the governing Eqs. (1) and (21), together with the boundary conditions, the wave dispersion equation can then be derived as (Dean and Dalrymple, 1984)

$$
\omega^{2}-g \tilde{k} \tanh \tilde{k} h=-i \omega \frac{k_{s}}{g}\left(g \tilde{k}-\omega^{2} \tanh \tilde{k} h\right) \tanh \tilde{k} d
$$

It is noted from (22) that only permeability $k_{s}$ has been taken into consideration in the wave dispersion relation.

\subsection{Consolidation model (C-M)}

If the problem is so slow that all acceleration forces are negligible, i.e. $\ddot{\vec{u}}$ and $\ddot{\vec{w}}$ vanish, the governing equations can then be simplified as:

$$
\left\{\begin{array}{l}
d \sigma_{i j}^{\prime}=D_{i j k l}\left(d \varepsilon_{k l}-d \varepsilon_{k l}^{0}\right) \\
\sigma_{i j, j}=0 \\
-\left(k_{i j} p_{, j}\right)_{, i}-\dot{\varepsilon}_{i i}=\frac{n_{e}}{K_{w}} \dot{p}
\end{array}\right.
$$


which leads to

$$
\left\{\begin{array}{l}
G\left\{\nabla^{2} \vec{u}+\frac{1}{1-2 \mu} \nabla(\nabla \cdot \vec{u})\right\}-\nabla p=0 \\
\frac{n_{e}}{K_{w}} \dot{p}+\nabla \cdot \dot{\vec{u}}-\frac{k_{s}}{\gamma} \nabla^{2} p=0
\end{array}\right.
$$

Zienkiewicz et al. (1980) developed a criterion to judge the loading speed for a periodic loading:

$$
\Pi_{1}=\frac{2 \rho k_{s}}{\pi \rho_{w} g} \frac{T}{T_{0}^{2}}, \quad \Pi_{2} \approx 10\left(\frac{T_{0}}{T}\right)^{2}
$$

where $T$ is the loading period; $T_{0}$ is the natural frequency of the soil, with $T_{0}=$ $2 L / V_{c}, \quad V_{c} \approx 1000 \mathrm{~m} / \mathrm{s} ; \Pi_{1}$ is applied to determining the drain situation and $\Pi_{2}$ to judging the dynamic effects of the bulk soil. According to criterion proposed by Zienkiewicz et al. (1980), the typical seabed problem should entirely neglect the dynamic effects. Thus, the consolidation model has been applied to the problems concerning soil dynamic response of seabed to water waves (Yamamoto et al., 1978; Madsen, 1978; Jeng, 1997). However, most previous investigations have considered the wave pressure penetrating into the seabed, rather than the wave damping. Furthermore, Zienkiewicz et al. (1980) only provide a one-dimensional analysis, which may not be able to represent the phenomenon of the wave-seabed interaction.

The solution of the governing eq. (23), together with boundary conditions (5) and (7), is rather familiar to us, so the wave dispersion relation can be written as:

$$
\omega^{2}-g \tilde{k} \tanh \tilde{k} h=\omega^{2}\left[i \omega u_{z}(0)-\frac{k_{s} \partial p(0)}{\gamma \partial z}\right] \sec (\tilde{k} h)
$$

where $u_{z}(0), p(0)$ can be obtained by applying the boundary conditions (5) and (7). It is noted that (25) can be further simplified to the form identical to the wave dispersion equation proposed by Jeng (2001).

\subsection{Coulomb-damping model (Y-M)}

Yamamoto (1983) developed a model considered the friction between grains (Coulomb-damping). The governing equations of motion of marine skeletal frame and pore fluid can be given as:

$$
\left\{\begin{array}{l}
\tilde{G} \nabla^{2} \vec{u}-(\tilde{H}-\tilde{G}) \nabla \varepsilon_{v}-\tilde{C} \nabla \zeta=\rho \ddot{\vec{u}}+\rho_{w} \ddot{w}^{\prime} \\
\nabla(\tilde{C} e-\tilde{M} \zeta)=\frac{\gamma n_{e} \dot{\vec{w}}^{\prime}}{k_{s}}+\left(\rho_{w} \ddot{\vec{u}}+m \ddot{\vec{w}}^{\prime}\right)
\end{array}\right.
$$


where $\tilde{G}=G(1+i \delta)$ is the complex shear modulus and given by the $\mathrm{G}$ and specific energy $\operatorname{loss} \delta ; \tilde{H}, \quad \tilde{C}$ and $\tilde{M}$ denote Biot's complex elastic moduli of the porous media and are derived by physical parameters of soil; the quantity $m=\frac{\rho_{w}}{n_{e}}(1+\alpha)$ is the virtual mass of soil skeletal frame in the accelerated flow field, $\alpha$ is the added mass coefficient of the skeletal frame; $\zeta$ is defined as: $\zeta=-\nabla \cdot \vec{w}^{\prime}=-n_{e} \nabla \cdot(\vec{w}-$ $\vec{u})$.

For the sake of convenience, we give an alternative form of (26):

$$
\left\{\begin{aligned}
\tilde{G}\left[\nabla^{2} \vec{u}\right. & \left.+\frac{1}{1-2 \mu} \nabla(\nabla \cdot \vec{u})\right]-\nabla p=n_{e} \rho_{w} \ddot{\vec{w}}+\left(1-n_{e}\right) \rho_{s} \ddot{\vec{u}} \\
& \quad-\tilde{M}(1-\varepsilon)\left\{\left(n_{e}-\varepsilon\right) \nabla(\nabla \cdot \vec{u})-n_{e} \nabla(\nabla \cdot \vec{w})\right\} \\
-\nabla p= & \rho_{w} \alpha \ddot{\vec{u}}+\rho_{w}(1+\alpha) \dot{\vec{w}}+\frac{\gamma n_{e}}{k_{s}}(\dot{\vec{w}}-\dot{\vec{u}}) \\
\frac{n_{e}}{K_{w}} \dot{p}+ & \nabla \cdot \dot{\vec{u}}-\frac{k_{s}}{\gamma} \nabla^{2} p-\frac{k_{s}}{\gamma} \rho_{w}(1+\alpha) \nabla \cdot \ddot{\vec{w}} \\
\quad & \frac{k_{s}}{\gamma} \rho_{w} \alpha \nabla \cdot \ddot{\vec{u}}+\frac{n_{e}}{K_{w}} \tilde{M}\left\{\left(n_{e}-\varepsilon\right) \nabla \cdot \dot{\vec{u}}-\left(n_{e}-\frac{\tilde{D} K_{w}}{n_{e} K_{r}^{2}}\right) \nabla \cdot \dot{\vec{w}}\right\}
\end{aligned}\right.
$$

where

$$
\begin{aligned}
& \tilde{M}=\frac{K_{r}^{2}}{K_{r}\left[1+n_{e}\left(K_{r} / K_{w}-1\right)\right]-\tilde{K}_{s}} \\
& \varepsilon=1-\tilde{K}_{s} / K_{r}
\end{aligned}
$$

and $K_{r}, \tilde{K}_{s}\left(=G\left(\frac{2 \mu}{1-2 \mu}+\frac{2}{3}\right)(1+i \delta)\right)$ is the bulk modulus of soil and soil skeletal frame, respectively.

Comparing (2) and (27), if $\alpha=0$ and $\tilde{M}=0$, equation (27) is equivalent to eq. (2). The real $(\tilde{M})$ is a measure of the amount of water which can be forced into the soil under pressure while the volume of the soil is kept constant, and $\varepsilon$ measures the ratio of water volume squeezed out of the volume change of the soil if the latter is compressed while allowing the water to escape. For an incompressible fluid and an incompressible matrix material, $\varepsilon=1, \tilde{M}=\infty$. Thus, the fluid pressure does not appear. It is obvious that the constant $\tilde{M}$ will be of significance for a soil not completely saturated with water and containing air bubbles. In such a case, the constants $\tilde{M}$ can take values depending on the degree of saturation of the soil. The model is paid more attention to the relative motion between soil and pore fluid. With the model, a series of studies have been carried out and some solutions have been applied 
to field measurements (Yamamoto and Takahashi, 1985; Yamamoto and Trevorrow, 1991).

In the Coulomb-damping model, the wave dispersion relation can be written as:

$$
\begin{aligned}
& \omega^{2}-g \tilde{k} \tanh (\tilde{k} h)=\left\{1-\left[\left(\tilde{a}_{1 f}-\tilde{a}_{2 f}\right)\left(1+\tilde{c}_{f}\right) \tilde{\lambda}_{f}+\left(\tilde{a}_{1 s}-\tilde{a}_{2 s}\right)\left(1+\tilde{c}_{s}\right) \tilde{\lambda}_{s}\right.\right. \\
& \left.\left.\quad-i \tilde{k}\left(\tilde{a}_{1 T}+\tilde{a}_{2 T}\right)\left(1+\tilde{c}_{T}\right)\right] \sec (\tilde{k} h)\right\}
\end{aligned}
$$

in which the complex parameters $\tilde{a}_{1,2(f, s, T)}, \tilde{c}_{f, s, T}, \tilde{\lambda}_{f, s}$ can be referred to the authors' previous papers (Lin, 2001; Lee et al., 2002b).

In general, the simplified formulation will be encouraged for more economical processing of the solution. Sometimes, however, the error can be accompanied by various limiting approximations. In the next section, we will discuss the wave attenuation with the numerical results of the four different models.

\section{Numerical results and discussions}

The objective of this study is to clarify the differences between the existing models (D-M, C-M and Y-M) and proposed model (Z-M model). In this section, we will examine wave-induced soil motion and pore fluid motion and wave damping ratio in the marine sediment with the aforementioned models. Then, the applicable range of models will be clarified for engineering practice. To achieve it, the input data of numerical examples are tabulated in Table 1.

\subsection{Soil motion and pore fluid motion}

As mentioned previously, there are two main terms in the wave dispersion relation. They are: soil motion $\left(u_{z}\right)$ and pore fluid motion $\left(w_{z}\right)$. Since the results from D-M

Table 1

Water wave and soil parameters

\begin{tabular}{lll}
\hline Name of soil parameter & Ranges of parameters \\
\hline Soil permeability, $\mathrm{k}_{\mathrm{s}}(\mathrm{m} / \mathrm{s})$ & $10^{-1}$ (gravel) & $10^{-2}$ (coarse sand) $10^{-4}$ (fine sand) \\
Shear modulus, $\mathrm{G}\left(\mathrm{N} / \mathrm{m}^{2}\right)$ & $5 \times 10^{7}$ (gravel) & $10^{7}$ (coarse sand) $5 \times 10^{6}$ (fine sand) \\
Poisson's ratio, $\mu$ & 0.3 & \\
Porosity, $\mathrm{n}_{\mathrm{e}}$ & 0.4 \\
Density of soil skeleton, $\rho_{\mathrm{s}}$ & $2650 \mathrm{~kg} / \mathrm{m}^{3}$ \\
Density of pore fluid, $\rho_{\mathrm{w}}$ & $1000 \mathrm{~kg} / \mathrm{m}^{3}$ \\
Thickness of seabed & $0.1 \mathrm{~L}_{\mathrm{d}}$ or various \\
\hline Name of water wave & Ranges of parameters \\
parameter & \\
\hline Wave period $\mathrm{T}(\mathrm{s})$ & 10.0 \\
Water depth, $\mathrm{h}(\mathrm{m})$ & 15 or various \\
\hline
\end{tabular}


and $\mathrm{C}-\mathrm{M}$ are identical in the soil motion and pore fluid motion, we only present the results of $\mathrm{C}-\mathrm{M}$ for the comparison. Fig. 1 illustrates the relationship between soil motion and relative water depth $\left(h / L_{d}\right)$ with different models. As shown in the figure, the soil motions obtained from C-M and Z-M are identical in coarser materials (such as gravel and coarse sand), while a significant difference between two models (C$\mathrm{M}$ and Z-M) is observed in fine sand. Fig. 1 also indicates that the soil motion obtained from Y-M model is greater than another two models. This implies that the inclusion of inertia term, $\ddot{u}_{i}$ is too small to be considered in coarser materials. Under such conditions, the Coulom-friction damping may be more important than inertia effects.

The distributions of the pore fluid motion vs. relative water depth with various models are also illustrated in Fig. 2. It is noted that the non-dimensional pore fluid motion, $2 G k_{0}\left|w_{z}\right| / p_{0}$, obtained from Z-M model is much greater than another two models (i.e. C-M and Y-M). This difference is more significant in fine sand, as
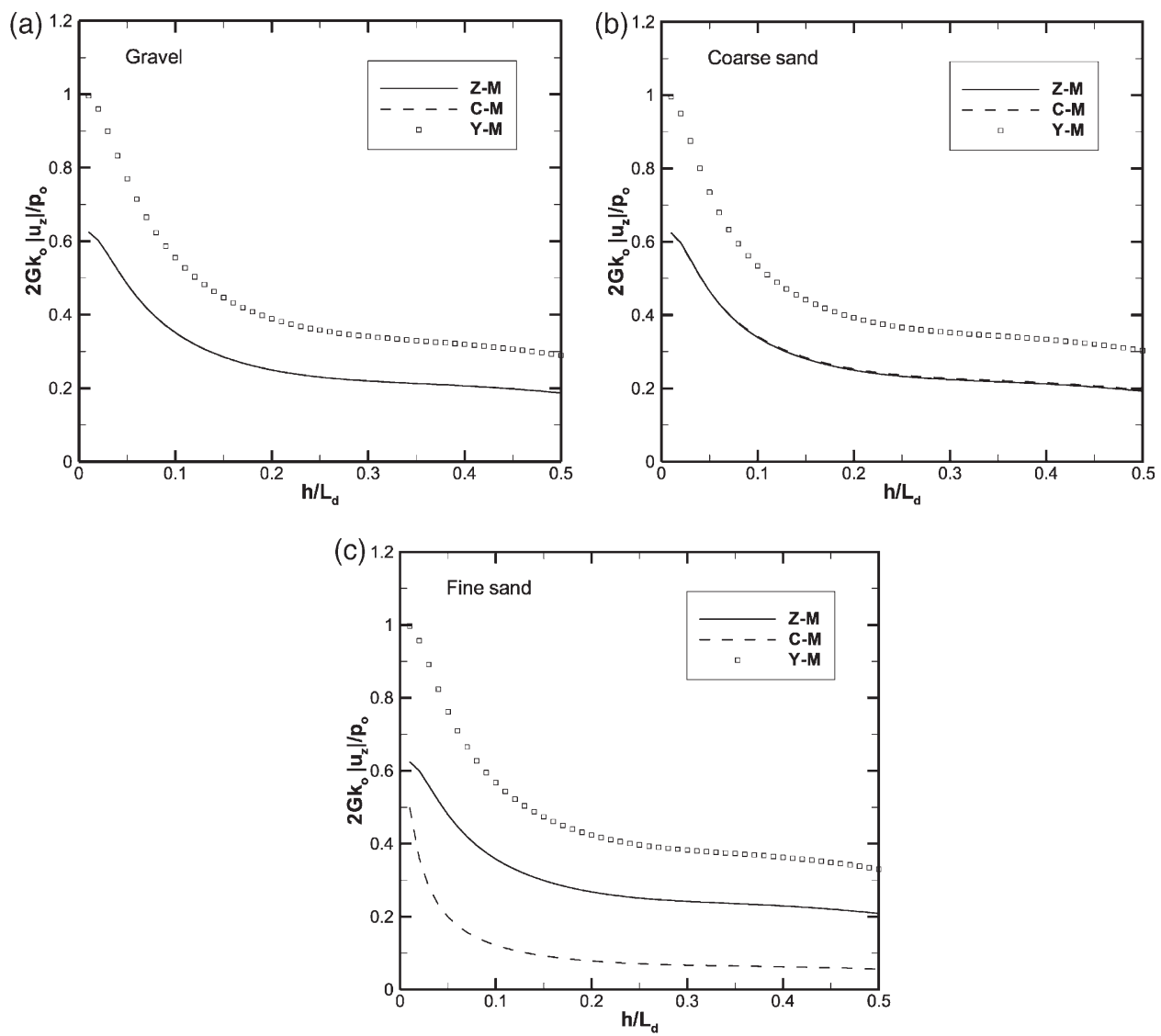

Fig. 1. Distribution of the soil motion vs. relative water depth $h / \mathrm{L}_{\mathrm{d}}$ in (a) gravel, (b) coarse sand, and (c) fine sand. 
(a)

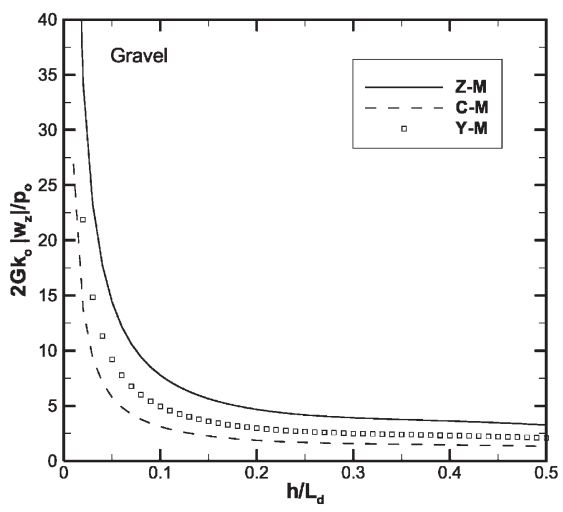

(b)

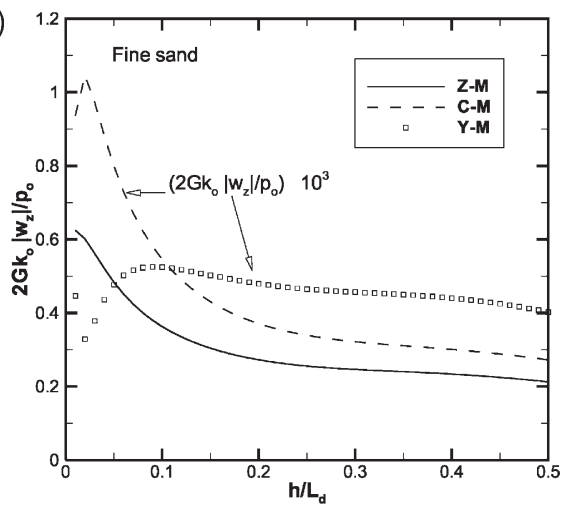

(c)

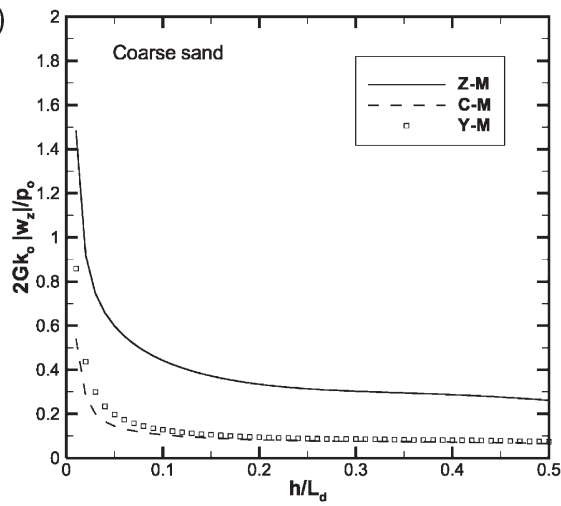

Fig. 2. Distribution of the pore fluid motion vs. relative water depth $h / L_{d}$ in (a) gravel, (b) coarse sand, and (c) fine sand.

shown in Fig. 2. The results indicate that the model concerning on interaction of soil skeletal frame and pore fluid restrain the defect due to the layer flow assumption of Darcy's law. Thus, we consider the Z-M model is more applicable in graveled seabed, while the Y-M provides a better estimation in fine sand.

\subsection{Wave damping in a seabed}

In this study, the complex wave number consists of two components. The real wave number is related to the wavelength, while the imagery component is the damping of water waves. Thus, it is common to use the ratio of two wave number components $\left(2 k_{\mathrm{i}} / k_{\mathrm{r}}\right)$ to represent the wave damping ratio. Fig. 3 illustrates the distribution of non-dimension wave damping $2 k_{\mathrm{i}} / k_{\mathrm{r}}$ vs. water depth $h / L_{\mathrm{d}}\left(L_{d}=1.56 \mathrm{~T}^{2}\right.$, which is the wavelength in deep water) in three different seabeds (gravel, coarse sand and fine sand). In the figures, two special cases, no-Coulomb friction $(\delta=0)$ and noadded mass $(\alpha=0)$, are also included. The figure clearly indicates that the wave damping decrease as the relative water depth $\left(h / L_{d}\right)$ increases. Furthermore, the dif- 

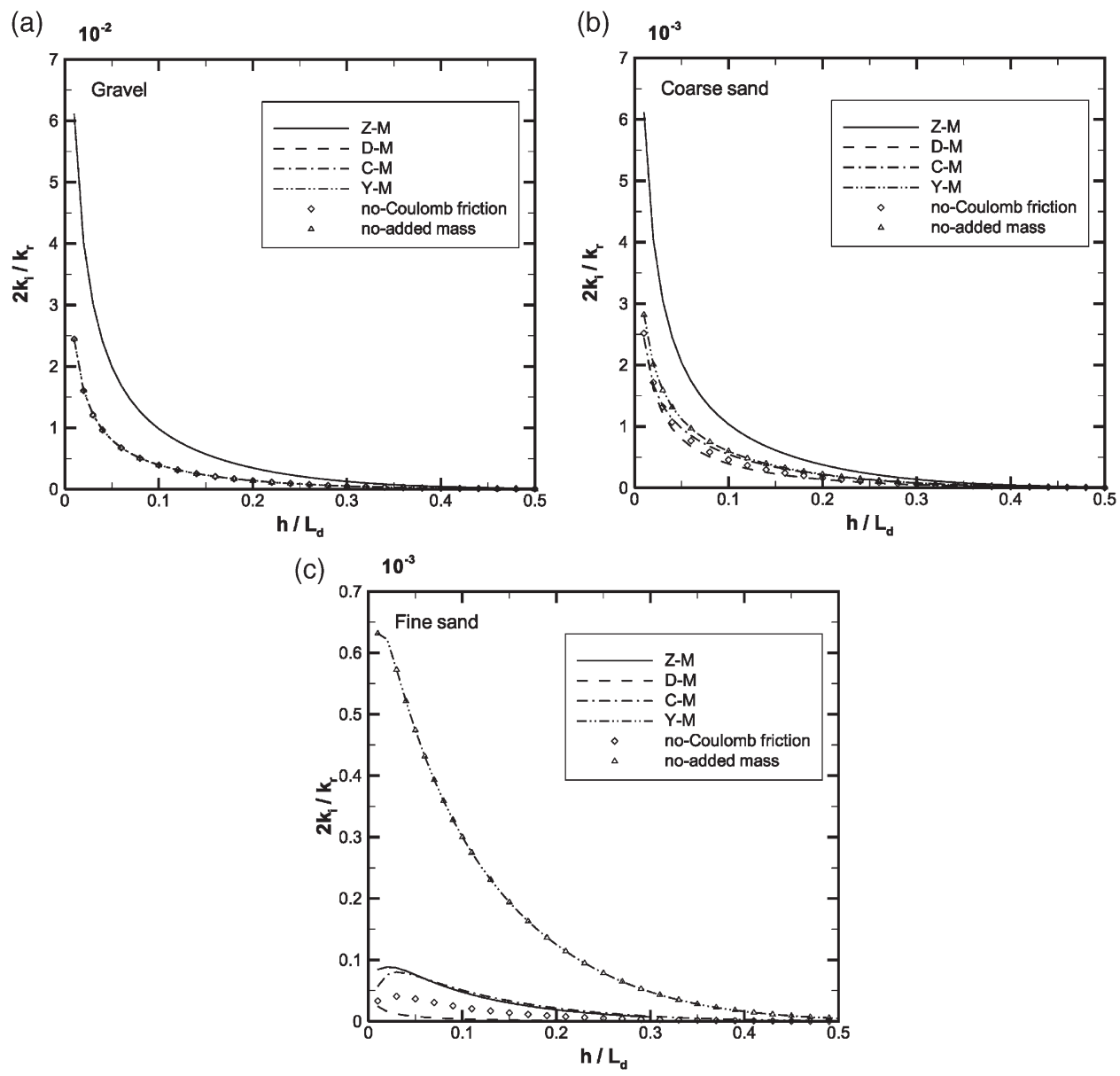

Fig. 3. Distribution of the damping ratio $2 \mathrm{k}_{\mathrm{i}} / \mathrm{k}_{\mathrm{r}}$ versus relative water depth $\mathrm{h} / \mathrm{L}_{\mathrm{d}}$ in (a) gravel, (b) coarse sand, and (c) fine sand.

ference among the models can be neglected as $\mathrm{h} / \mathrm{L}_{d}>0.4$. For the gravel seabed (Fig. 3(a)), the damping ratio is the same of the D-M, C-M and Y-M, and ratio from $\mathrm{Z}-\mathrm{M}$ is much larger than other models in shallow water case. As the shear modulus and the permeability of seabed decrease, the results obtained by each model appear diversified (Fig. 3(b) and 3(c)). It is also noted that the difference between models increases in the softer seabed, comparing Fig. 3(a)-(c).

Fig. 3 also indicates that the influence of added mass on wave damping is very small, while the influence of Coulomb friction is significant, especially for fine sand. This is due to the function of Coulomb friction between the grains become more important as the size of grain is small. Meanwhile, for the fine sand seabed, the damping ratio obtained by $\mathrm{Z}-\mathrm{M}$ and $\mathrm{C}-\mathrm{M}$ are approaching. This implies that the action of inertia term is reduced, when the seabed become more pliant. 
The opposite trend is observed from Fig. 4, which indicates further that wave damping ratio varies with the relative seabed thickness $\left(d / L_{d}\right)$. That is, the difference increases as $d / L_{d}$, increases. However, the difference among the models increases as $d / L_{d}$ increases, too.

In summary, the damping ratio obtained from Z-M model is always greater than others in gravel, even for coarse sand. The damping ratios obtained from D-M and $\mathrm{C}-\mathrm{M}$ models are almost identical in a fully saturated seabed. However, the damping ratio from Y-M model is greater than others in fine sand seabed. It is also concluded that not only the Coulomb friction but also all acceleration forces (i.e. inertia terms) affect the wave damping.

(a)

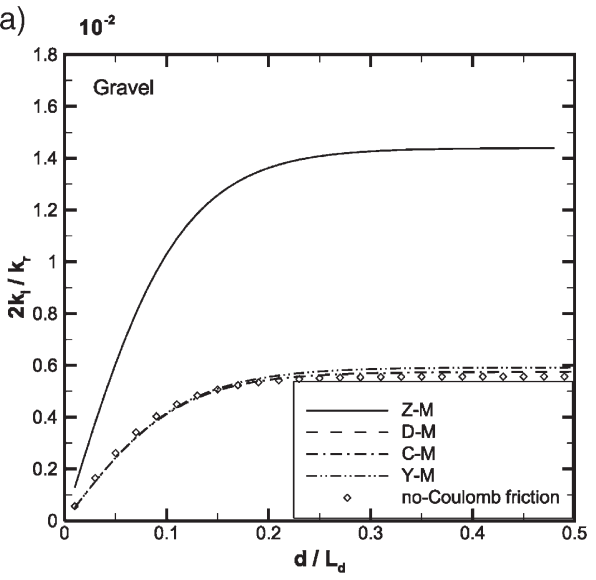

(b)

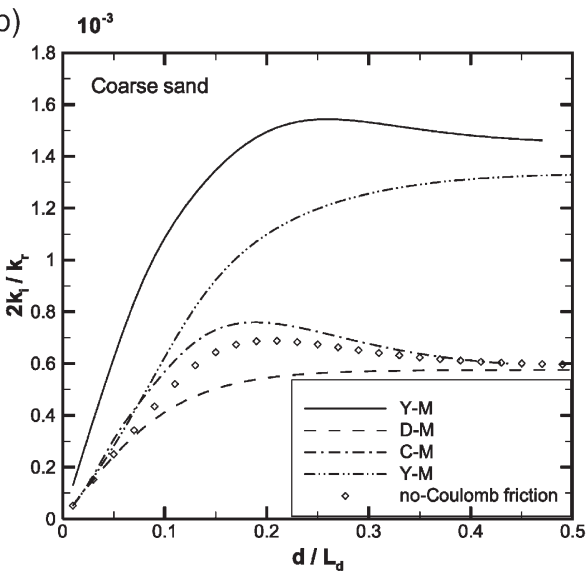

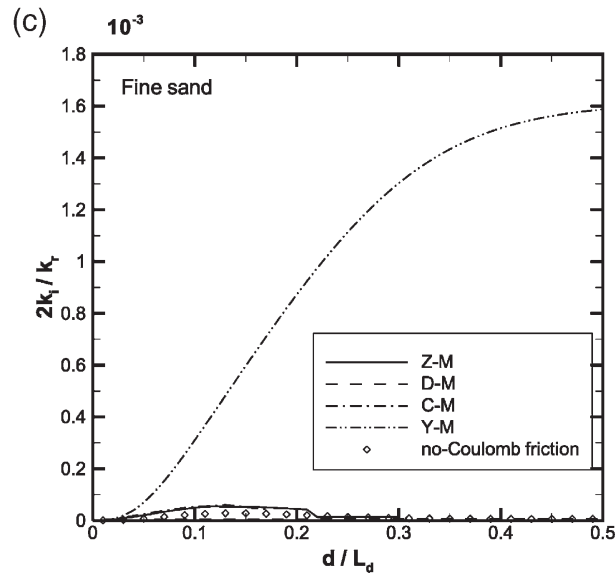

Fig. 4. Distribution of the damping ratio $2 k_{i} / k_{r}$ versus relative seabed thickness $d / L_{d}$ in (a) gravel, (b) coarse sand, and (c) fine sand. 


\subsection{Application of different models}

Extrapolating from the above discussions, there are four parameters influencing wave attenuation, i.e. the soil permeability, shear modulus, seabed thickness and water depth. Thus, we will try to search the applicable zones for each model, as follows.

According to dimensional analysis, we define two non-dimensional parameters:

$$
\theta_{1}=\frac{K_{f} k_{s} \rho_{w} k_{d}}{G \omega}, \quad \theta_{2}=\frac{d}{h}
$$

where $K_{\mathrm{f}}$ is the bulk modulus of pore fluid, which is taken as $10^{9} \mathrm{~N} / \mathrm{m}^{2}$. Considering a typical sand seabed, in which the permeability varies between $10^{-4} \mathrm{~m} / \mathrm{s}$ and $10^{-1}$ $\mathrm{m} / \mathrm{s}$, shear modulus $G=10^{7} \mathrm{~N} / \mathrm{m}^{2}$, seabed thickness $d=10^{-3} L_{d} \sim 0.4 L_{d}$, water wave period $T=10 \mathrm{~s}$, water depth $h=0.1 \mathrm{~L}_{d}$. With this input data, we see that the range of the parameters is: $1<\theta_{1}<10^{3}, 0.01<\theta_{2}<4.0$.

As shown in Fig. 5, the damping ratio obtained from Z-M model ranges from $2.5 \times 10^{-6} \sim 2 \times 10^{-2}$ with various values of $\theta_{1}$. Similarly, the damping ratio obtained from $\mathrm{C}$-M model varies between $2.3 \times 10^{-6} \sim 8.5 \times 10^{-3}$, while it varies between $1.1 \times 10^{-6} \sim 9.5 \times 10^{-3}$ for the Y-M model. The figure also indicates the influence of $\theta_{2}$ on the damping ratio. The figure clearly indicates that the influences of $\theta_{2}$ on the damping ratio is insignificant, when $\theta_{2}$ is less than 0.5 .

Comparing sub-figures in Fig. 5, it is found that, as $\theta_{1}<100$, the curves by Z$\mathrm{M}$ and $\mathrm{C}-\mathrm{M}$ are approaching and the curves at $\mathrm{Z}-\mathrm{M}$ and $\mathrm{Y}-\mathrm{M}$ intersect at some points. Here, we proposed a relation for the applicability zone of the C-M, that is: $\mid 2 k_{i} / k_{r}($ for $Z-M)-2 k_{i} / k_{r}($ for $C-M$ or $D-M) \mid<5 \times 10^{-5}$

If the above relation is satisfied, we consider that the $\mathrm{Z}-\mathrm{M}$ can be replaced by $\mathrm{C}$ $\mathrm{M}$ (or D-M). By the sp-line method, the summary of the conclusion taken from the examination of many computational results is shown in Fig. 6. In the figure, the zone of applicability of each model has been clearly indicated.

\section{Conclusion}

Water wave attenuation due to the seabed is important for both coastal and geotechnical engineering. In this paper, a detailed analysis of full dynamic response of seabed to water waves is derived. Based on numerical examples presented, it is found that in most situations the inertia terms should not be omitted as $\mathrm{h} / \mathrm{L}_{d}<0.5$. For a gravel seabed, the proposed full dynamic model (i.e. Z-M model) should be used, while for fine sand seabed the Y-M model is viable. Finally, two non-dimensional parameters have been introduced and a criterion is given. According to the criterion, the applicability of each model has been clarified.

In the foregoing discussion, we concentrated on the wave damping for saturated seabed. In natural marine sediment, however, tiny air pockets are entrained in the pores, and the mixture of air and water has a relatively high compressibility, therefore 

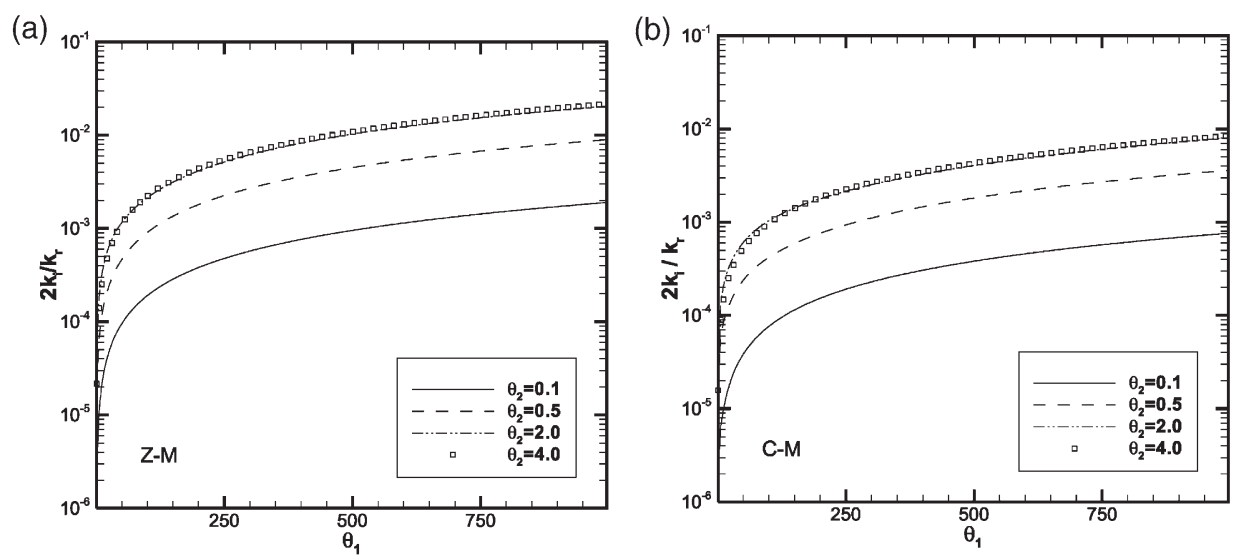

(c)

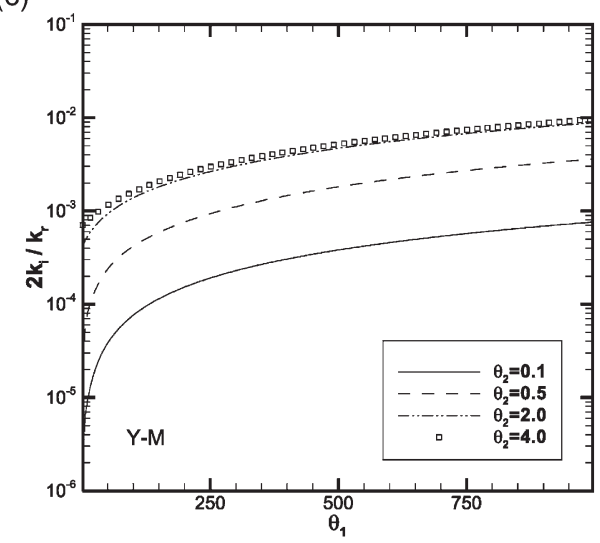

Fig. 5. Distribution of the damping ratio versus non-dimensional parameter $\theta_{1}$ for various $\theta_{2}$ with different models. (a) $\mathrm{Z}-\mathrm{M}$ model, (b) $\mathrm{C}-\mathrm{M}$ model and (c) $\mathrm{Y}-\mathrm{M}$ model.

the effect of degree of saturated and the stresses distribution in the seabed for the models should be involved in future studies.

\section{Acknowledgements}

We appreciate the comments of Professor J.R. Chaplin. The first author, Dr. Lin is grateful to the Royal Society for providing a Fellowship. This work was financially supported by NSFC funds (40176027). The second author, Dr. Jeng, is grateful to the Australian Research Council for the financial supports under ARC Large Grant Scheme (A00104092). 


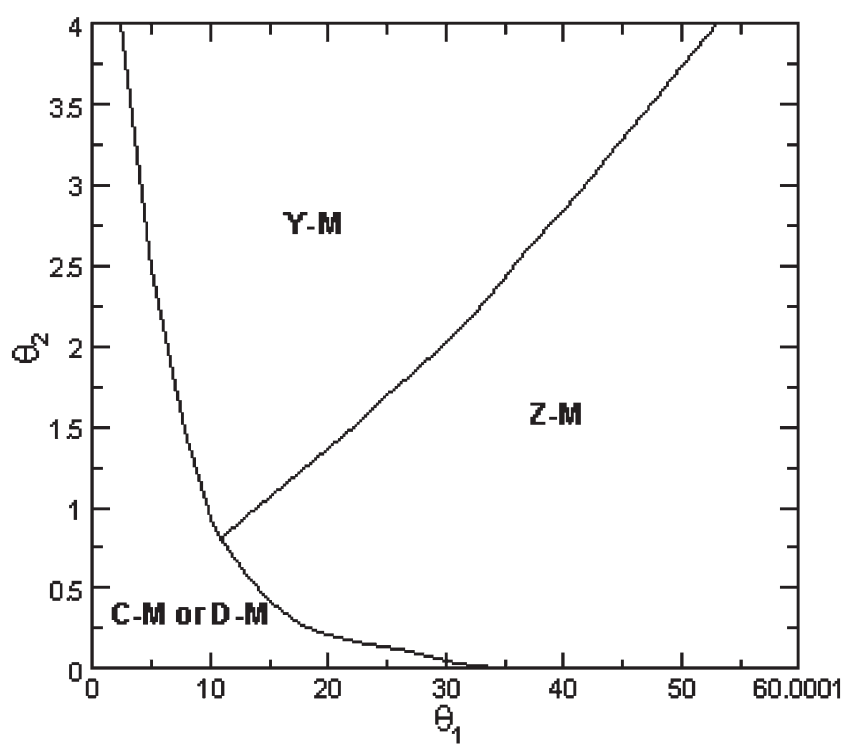

Fig. 6. Applicable ranges of the existing poro-elastic models for wave-seabed interaction.

\section{References}

Biot, M.A., 1956. Theory of propagation of elastic waves in a fluid-saturated porous solid: I. Low frequency range. The Journal of Acoustical Society of America 28 (2), 168-178.

Dalrymple, R.A., Liu, P.L.F., 1978. Waves over soft mud: a two-layer fluid model. Journal of Physical Oceanography 8, 1121-1130.

Dean, R.G., Dalrymple, R.A., 1984. Water Wave Mechanics for Engineers and Scientists. Prentice-Hall International, London.

Gade, H., 1958. Effects of a non-rigid impermeable bottom on plane surface waves in shallow water. Journal of Marine Research 16, 61-82.

Hsiao, S.V., Shemdin, O.H., 1980. Interaction of ocean waves soil bottom. Journal of Physical Oceanography 10, 605-610.

Jeng, D.S., 1997. Wave-induced seabed response in front of a breakwater. PhD Thesis, The University of Western Australia, 297 pp.

Jeng, D.S., 2001. Wave dispersion equation in a porous seabed. Ocean Engineering 28, 1585-1599.

Kim, M.H., Koo, W.C., Hong, S.Y., 2000. Wave interactions with 2D structures on/inside porous seabed by a two-domain boundary element method. Applied Ocean Research 22 (5), 255-266.

Lee, T.L., Tsai, C.P., Jeng, D.S., 2002a. Analytical solution of wave-induced seabed response: effects of inertia forces. Ocean Engineering 29 (12), 1577-1601.

Lee, T.L., Tsai, C.P., Jeng, D.S., 2002b. Ocean waves propagating over a coulomb-damped poroelastic seabed of finite thickness: An analytical solution. Computers and Geotechnics 29 (2), 119-149.

Lin, M., 2001. The Analysis of silt behaviour induced by water waves. Science in China (E) 31 (1), 86-96.

Liu, P.L.F., 1973. Damping of water waves over porous bed. Journal of Hydraulics Division ASCE 99, 2263-2271.

Macpherson, H.J., 1980. The attenuation of water waves over a non-rigid bed. Journal of Fluid Mechanics 97, 721-730.

Madsen, O.S., 1978. Wave-induced pore pressures and effective stresses in a porous bed. Geotechnique 28 (4), 377-393. 
Reid, R.O., Kajiura, K., 1957. On the damping of gravity waves over a permeable seabed. Trans Am Geophysical Union 38, 939-950.

Sawaragi, T., Deguchi, I., 1992. Wave on permeable layers. Proceedings of the 23rd International Conference on Coastal Engineering, ASCE, pp. 1531-1544.

Yamamoto, T., 1983. On the response of a Coulomb-damped poro-plastic bed to water waves. Marine Geotechnology 5 (2), 93-130.

Yamamoto, T., Koning, H.L., Sellmejjer, H., Hijum, E.V., 1978. On the response of a poro-elastic bed to water waves. Journal of Fluid Mechanics 87, 193-206.

Yamamoto, T., Takahashi, S., 1985. Wave damping by soil motion. Journal of Waterway, Port and Ocean Engineering, ASCE 111, 62-77.

Yamamoto, T., Trevorrow, M., 1991. Experimental verifications of bottom shear modulus profiler (BSMP) method. Proceedings of the International Conference on Geotechnical Engineering for Coastal Development Theory and Practice on Soft Ground (Geot-Coastal 91), Yokohama, Japan, 1, 123-128.

Zienkiewicz, O.C., Chang, C.T., Bettess, P., 1980. Drained, undrained, consolidating and dynamic behaviour assumptions in soils. Geotechnique 30 (4), 385-395. 